\title{
Quantum dynamics in potentials with fast spatial oscillations
}

\author{
Viktor Novičenko, ${ }^{*}$ Julius Ruseckas, $^{\dagger}$ and Egidijus Anisimovas ${ }^{\ddagger}$ \\ Institute of Theoretical Physics and Astronomy, Vilnius University, Sauletekio Ave. 3, LT-10257 Vilnius, Lithuania
}

(Dated: March 14, 2019)

\begin{abstract}
We consider quantum dynamics of systems with fast spatial modulation of the Hamiltonian. Employing the formalism of supersymmetric quantum mechanics and decoupling fast and slow spatial oscillations we demonstrate that the effective dynamics is governed by a Schrödinger-like equation of motion and obtain the expression of the resulting effective Hamiltonian. In particular, we show that there exists an attractive effective potential even in the case when the oscillating potential averages to zero.
\end{abstract}

\section{INTRODUCTION}

The idea to simplify the analysis of a physical problem by taking into account the presence of substantially different characteristic scales (spatial, temporal or other) is a ubiquitous and powerful one. Far-reaching examples include the Born-Oppenheimer approach [1, 2], based on decoupling of fast and slow vibration modes, and Floquet engineering [3], which fruitfully exploits the idea to realize effective Hamiltonians with desired properties by applying a time-periodic driving to a controllable physical system [4]. In the limit when the periodic driving sets the dominant frequency scale (in comparison to the internal dynamics of the system) the resulting (stroboscopic) dynamics is described by the time-averaged driven Hamiltonian. Already this rather simple result has led to numerous insights and ground-breaking experimental schemes [5-10]. When the driving sets the largest but not overwhelming frequency scale, the resulting dynamics may be captured by a systematic inverse-frequency expansion [11-13], which also offers opportunities for quantum engineering of physically interesting model Hamiltonians $[14,15]$

In the present contribution, we look at the complementary facet of spatial, rather than temporal, modulation of the Hamiltonian. We show that in the limit of rapid oscillations of the potential, the effective dynamics is governed by a Schrödinger-like equation of motion. The main contribution to the effective potential featured in this equation is proportional to the square of the envelope function that modulates the rapid oscillations of the true potential. Moreover, this contribution comes with a negative sign, i.e. there exists an attractive effective potential even when the oscillating potential averages to zero. The effective Schrödinger equation is solved by a smoothed wave function which accurately approximates the overall shape of the true wave function but excludes its rapid smallscale oscillations. The obtained description is relevant to describe quantum dynamics in potentials formed by interfering laser beams. Here, the resulting intensity dis-

\footnotetext{
* viktor.novicenko@tfai.vu.lt; http://www.itpa.lt/〜 novicenko/

$\dagger$ julius.ruseckas@tfai.vu.lt; http://web.vu.lt/tfai/j.ruseckas/

¥ egidijus.anisimovas@ff.vu.lt
}

tributions, which define the potentials felt by ultracold atoms, typically combine rapid variations on the scale of the wavelength with slow modulation due to the shape of the beams [16].

Interestingly, in the derivation of the effective Schrödinger equation we benefited from an approach based on supersymmetric quantum mechanics (SUSY QM). SUSY QM is a generalization of the factorization method commonly used for the harmonic oscillator. It was first introduced as a model to study non-perturbative symmetry breaking in supersymmetric field theories [17]. Later it was realized that SUSY QM is an interesting field in its own right, and the ideas of supersymmetry have been profitably applied to many quantum mechanical problems; see Refs. [18-20] for books and reviews.

Our paper is organized as follows: In Sec. II we derive the expression for the effective potential in a system with fast spatial potential modulation. To verify the validity of the effective Hamiltonian, in Sec. III we compare numerically calculated eigenfunctions of the original Hamiltonian with the eigenfunctions obtained using the effective Hamiltonian. In Sec. IV we discuss applications to potentials for ultracold atoms formed by interfering laser beams. Finally, in Sec. V we summarize our findings.

\section{DYNAMICS IN A SPATIALLY MODULATED POTENTIAL}

We study the motion of a one-dimensional quantum particle of mass $m$ in a static spatially modulated potential $V(x)$ whose shape can be represented as a combination of a slowly varying envelope function $\Phi(x)$ with vanishing limiting values

$$
\lim _{x \rightarrow \pm \infty} \Phi(x)=0
$$

and a periodic function $v(k x)=v(k x+2 \pi)$. The period of the rapid oscillations of the potential is given by $2 \pi / k$ and by assumption must be much smaller than the characteristic length scale of the envelope function $\Phi(x)$. The dynamics of the particle is described by the Schrödinger equation

$$
i \hbar \frac{\partial}{\partial t} \psi=\hat{H} \psi
$$


with the Hamiltonian

$$
\hat{H}=-\frac{\hbar^{2}}{2 m} \frac{\partial^{2}}{\partial x^{2}}+V(x) .
$$

Our goal is to derive an effective Hamiltonian that approximates the ensuing dynamics for large but finite $k$, and becomes exact in the limit $k \rightarrow \infty$. The potentialenergy term is taken of the form

$$
V(x)=k v(k x) \Phi(x)+\rho(x) .
$$

Here, $v(s)$ is a periodic function that averages to zero over a period

$$
\langle v\rangle=\frac{1}{2 \pi} \int_{0}^{2 \pi} v(s) \mathrm{d} s=0
$$

and in Eq. (4) is additionally scaled by a factor $k$, that is, the increasing frequency of spatial oscillations is complemented by increasing amplitude. As further explained below, if this was not done, in the limit $k \rightarrow \infty$ the oscillatory potential $v(k x) \Phi(x)$ would average out. The presence of a finite potential background is taken into account by a separate term $\rho(x)$, which is also required to vanish for $|x| \rightarrow \infty$, cf. Eq. (1). To be able to work with dimensionless quantities, we identify a characteristic length scale $\ell$ and measure coordinates in units $\ell$, wavenumbers in $\ell^{-1}$, energies in units $\hbar^{2} /\left(2 m \ell^{2}\right)$ and time in $2 m \ell^{2} / \hbar$. The dimensionless Schrödinger equation reads

$$
i \frac{\partial}{\partial t} \psi=\left\{-\frac{\partial^{2}}{\partial x^{2}}+k v(k x) \Phi(x)+\rho(x)\right\} \psi(x, t) .
$$

We note that a related problem, as a specific case, was studied in Ref. [21], where ponderomotive dynamics was derived as an expansion with respect to the inverse wavenumber $k^{-1}$. However, here we are interested in the regime of large oscillation amplitude [in comparison to the particle recoil energy $\left.\hbar^{2} /\left(2 m \ell^{2}\right)\right]$. This regime can not be directly covered by the formalism developed in Ref. [21].

\section{A. Derivation of the effective Hamiltonian using SUSY QM formalism}

We derive the effective Hamiltonian using an approach based on the formalism of SUSY QM (see for example [18]) and the subsequent application of the 'averaging' theorem [22, 23], which is a versatile tool that allows to eliminate rapidly oscillating terms in broad classes of first-order differential equation sets. (An alternative derivation is included as an Appendix.) To proceed, we write the Hamiltonian in a factorized form,

$$
\hat{H}=\hat{A}^{\dagger} \hat{A}
$$

where

$$
\hat{A}=\frac{\partial}{\partial x}+W(x)
$$

and we introduced the superpotential $W(x)$ as a solution of the differential equation

$$
\frac{d}{d x} W(x)=W^{2}(x)-k v(k x) \Phi(x)-\rho(x),
$$

known as the Riccati equation. Then we can replace the Schrödinger equation (6) by a pair of first-order differential equations

$$
\begin{aligned}
& \frac{\partial}{\partial x} \psi(x, t)=\varphi(x, t)-W(x) \psi(x, t), \\
& \frac{\partial}{\partial x} \varphi(x, t)=-i \frac{\partial \psi(x, t)}{\partial t}+W(x) \varphi(x, t),
\end{aligned}
$$

which govern the wave function $\psi(x, t)$ and an auxiliary function $\varphi(x, t)=\hat{A} \psi(x, t)$.

To be fully compliant with the requirements of the averaging theorem one should replace the partial derivatives $\partial / \partial x$ with the full derivatives $d / d x$. Formally, this can be done by discretizing the time interval into small steps of duration $\Delta t$, and introducing the notation $\psi_{(m)}(x) \equiv \psi(x, m \Delta t)$ and $\varphi_{(m)}(x) \equiv \varphi(x, m \Delta t)$. Then the term $\partial \psi(x, t) / \partial t$ can be approximated by a finite difference $\partial \psi(x, m \Delta t) / \partial t \approx\left[\psi_{(m+1)}-\psi_{(m)}\right] / \Delta t$. As a consequence, Eqs. (10) turn into set of differential equations for the functions $\psi_{(m)}(x)$ and $\varphi_{(m)}(x)$ formulated in terms of full derivatives with respect to the single variable $x$. However, to keep the notation simple we retain the partial derivatives having in mind that their presence poses no practical problems.

The three functions $W, \psi$ and $\varphi$ comprise the new dynamical variables and are governed by the set of firstorder nonlinear differential equations (9) and (10). Before proceeding to the averaging procedure, let us review the scaling of the potential energy, which we choose to write as $k v(k x) \Phi(x)$. At this stage we are in a position to see that - in the absence of the additional scaling by the factor $k$ - the term $v(k x) \Phi(x)$ would be eliminated from Eq. (9) by the averaging procedure as a rapidly oscillating term. In the presence of the scaling factor $k$, the potential energy $k v(k x) \Phi(x)$ becomes formally divergent in the $k \rightarrow \infty$ limit and must be cast into a manageable form. To achieve this aim, we replace $W$ with a new variable

$$
W^{\prime}=W+g(k x) \Phi(x)
$$

where the function $g(s)$ is the zero-average antiderivative of the function $v(s)$, i.e.,

$$
g(s)=\int_{0}^{s} v\left(s^{\prime}\right) d s^{\prime}-\frac{1}{2 \pi} \int_{0}^{2 \pi} \int_{0}^{s^{\prime \prime}} v\left(s^{\prime}\right) d s^{\prime} d s^{\prime \prime} .
$$

The purpose of the transformation from $W$ to $W^{\prime}$ can be elucidated by differentiating both sides of Eq. (11) with the result

$$
\frac{d}{d x} W+k v(k x) \Phi(x)=\frac{d}{d x} W^{\prime}-g(k x) \frac{d}{d x} \Phi(x),
$$


demonstrating the absorbtion of the term proportional to $k$. Thus, the new variables $W^{\prime}, \psi$, and $\varphi$ obey the differential equations

$$
\begin{aligned}
\frac{d W^{\prime}}{d x} & =\left[W^{\prime}-g(k x) \Phi(x)\right]^{2}-\rho(x)+g(k x) \frac{d \Phi}{d x} \\
\frac{\partial \psi}{\partial x} & =\varphi-\left[W^{\prime}-g(k x) \Phi(x)\right] \psi \\
\frac{\partial \varphi}{\partial x} & =-i \frac{\partial \psi}{\partial t}+\left[W^{\prime}-g(k x) \Phi(x)\right] \varphi .
\end{aligned}
$$

Here we consider continuous envelope functions $\Phi(x)$ so that the spatial derivative of the envelope represented by the term $d \Phi / d x$ in equation (14a) remains finite. The more general case of piecewise continuous envelope functions is treated in the following Subsection II B.

Now one can apply the averaging theorem [22, 23]. In general, the averaging theorem can be formulated as follows: Let us consider the vector $\mathbf{Z}(x)$ obeying the differential equation of the form

$$
\frac{d \mathbf{Z}}{d x}=\mathbf{F}(\mathbf{Z}, k x, x, k)
$$

where the vector field $\mathbf{F}$ is $2 \pi$-periodic with respect to the second argument, slowly depends (in comparison to the characteristic period $2 \pi / k$ ) on third argument and additionally depends on parameter $k$ represented by the fourth argument. One can introduce the averaged vector field as

$$
\overline{\mathbf{F}}(\mathbf{Z}, x)=\frac{1}{2 \pi} \int_{0}^{2 \pi}\left[\lim _{k \rightarrow \infty} \mathbf{F}(\mathbf{Z}, \vartheta, x, k)\right] d \vartheta .
$$

According to the averaging theorem, the solution $\overline{\mathbf{Z}}$ of the differential equation

$$
\frac{d \overline{\mathbf{Z}}}{d x}=\overline{\mathbf{F}}(\overline{\mathbf{Z}}, x)
$$

with the identical initial conditions $\mathbf{Z}\left(x_{0}\right)=\overline{\mathbf{Z}}\left(x_{0}\right)$ approximates the original solution $\mathbf{Z}(x)=\overline{\mathbf{Z}}(x)+\mathcal{O}\left(k^{-1}\right)$. Thus from Eqs. (14) one finds

$$
\begin{aligned}
\frac{d \overline{W^{\prime}}}{d x} & ={\overline{W^{\prime}}}^{2}+\left\langle g^{2}\right\rangle \Phi^{2}(x)-\rho(x) \\
\frac{\partial \bar{\psi}}{\partial x} & =\bar{\varphi}-\overline{W^{\prime}} \bar{\psi} \\
\frac{\partial \bar{\varphi}}{\partial x} & =-i \frac{\partial \bar{\psi}}{\partial t}+\overline{W^{\prime}} \bar{\varphi}
\end{aligned}
$$

where the averaged functions approximate the original functions as

$$
\left\{\overline{W^{\prime}}, \bar{\psi}, \bar{\varphi}\right\}=\left\{W^{\prime}, \psi, \varphi\right\}+\mathcal{O}\left(k^{-1}\right) .
$$

We note here, that the use of two distinct notations for 'averaging' is justified by their distinct meanings: the brackets $\langle v\rangle$ refer to the mean value of a periodic function $v$ taken over its period, whereas the overline $\bar{\psi}$ refers to a smoothed approximation of an oscillatory function, cf. Fig. 1.

Finally, transforming Eqs. (18) back to a single secondorder equation for the smoothed function $\bar{\psi}$, we obtain

$$
i \frac{\partial}{\partial t} \bar{\psi}=-\frac{\partial^{2}}{\partial x^{2}} \bar{\psi}+V_{\text {eff }}(x) \bar{\psi}
$$

with

$$
V_{\text {eff }}(x)=-\left\langle g^{2}\right\rangle \Phi^{2}(x)+\rho(x) .
$$

This result shows that the dynamics is governed by an effective equation of motion which retains the form of the usual Schrödinger equation with the effective Hamiltonian

$$
\hat{H}_{\mathrm{eff}}=-\frac{\partial^{2}}{\partial x^{2}}+V_{\mathrm{eff}}(x)
$$

where the effective potential (21) includes an attractive contribution proportional to the square of the envelope function. In the hindsight, this is intuitively clear: if there is no background, i.e. $\rho(x)=0$, the sign of the envelope function has no effect and only even powers of $\Phi(x)$ can contribute. Finally, let us stress that even though the wave function $\bar{\psi}$ approximates the original wave-function $\psi$ with the same accuracy as (19), i.e. $\bar{\psi}=\psi+\mathcal{O}\left(k^{-1}\right)$, an analogous statement does not hold for either the kinetic energy or the potential energy calculated using $\bar{\psi}$. Only the total energy is well approximated by the quantum-mechanical expectation value of the effective Hamiltonian with the smoothed wave function $\bar{\psi}$.

\section{B. Effective potential for piecewise continuous envelope}

Discontinuous envelope functions do not satisfy the requirement that the characteristic length scale of the envelope function should be much larger than the period of the rapid oscillations. Thus the effective potential (21) is valid only for continuous envelope functions $\Phi(x)$. In this subsection we will generalize our approach to include the case when the envelope is a piecewise continuous function. In this situation, the averaging is still applicable, however, at the points of discontinuity the smoothed functions $\bar{\psi}$ must obey appropriate boundary conditions.

For each coordinate $x$ which is not a point of discontinuity, all of the steps (9)-(18) can be repeated in exactly the same way as before. However, the points of discontinuity should be considered separately. At every point of discontinuity $x_{0}$ the superpotential $W(x)$ must remain continuous for any value of $k$ [cf. Eq. (9)]. Using Eq. (11) for the transformed superpotential $W^{\prime}$ we obtain

$$
\lim _{\varepsilon \rightarrow+0}\left[W^{\prime}\left(x_{0}+\varepsilon\right)-W^{\prime}\left(x_{0}-\varepsilon\right)\right]=g\left(\varphi_{0}\right) \Delta \Phi\left(x_{0}\right),
$$

where $\varphi_{0}=k x_{0}$ is the phase of the oscillating function $g(k x)$ at the point of discontinuity $x_{0}$ and $\Delta \Phi\left(x_{0}\right)=$ 


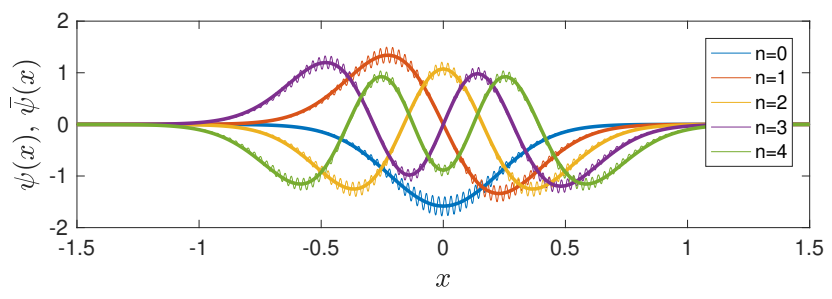

FIG. 1. (Color online) The five lowest-energy states calculated numerically using the original Hamiltonian (6) (thin oscillating curves) and found analytically for the effective Hamiltonian (25) (thick curves). The ground-state wave function is nodeless, and the excited states can be identified by the number of nodes. The parameters are set to $k=250$ and $a=2 \sqrt{210}$ such that $\lambda=20$ is an integer number.

$\lim _{\varepsilon \rightarrow+0}\left[\Phi\left(x_{0}+\varepsilon\right)-\Phi\left(x_{0}-\varepsilon\right)\right]$. This result indicates that at each point of discontinuity the transformed superpotential exhibits a step given by the product of the step of the envelope function and the local value of the periodic antiderivative $g(k x)$. As a special case, the transformed superpotential may remain continuous if $g\left(k x_{0}\right)=0$.

We must now require that $k$ grows to infinity in discrete steps, i.e. by assuming a sequence of monotonically growing values for which the phase $\varphi_{0}$ remains the same (modulo $2 \pi$ ). Then the condition (23) will also hold for averaged superpotential $\overline{W^{\prime}}(x)$, and can be satisfied by including terms proportional to the Dirac delta function $\Delta \Phi\left(x_{0}\right) g\left(\varphi_{0}\right) \delta\left(x-x_{0}\right)$ to the equation (18a). If there are several points of discontinuity $\left\{x_{n}\right\}$ and it is possible to find values of $k$ that keep the phases $\varphi_{n}$ constant, the effective potential (21) becomes

$$
\begin{aligned}
V_{\mathrm{eff}}(x)= & -\left\langle g^{2}\right\rangle \Phi^{2}(x)+\rho(x) \\
& -\sum_{n} \Delta \Phi\left(x_{n}\right) g\left(\varphi_{n}\right) \delta\left(x-x_{n}\right) .
\end{aligned}
$$

We see that step discontinuities in the envelope function translate into delta-function singularities in the effective potential. As a special case, these singularities may be absent, if $g\left(\varphi_{n}\right)=0$.

\section{NUMERICAL EXAMPLES}

To verify the validity of the effective description we perform a numerical calculation of the eigenfunctions for the original Hamiltonian (6) and compare them with the eigenfunctions obtained using the effective Hamiltonian (20). We treat both cases of continuous and piecewise continuous envelope functions and use the effective potentials given, respectively, by Eq. (21) and Eq. (24).

Starting with the simpler case of a smooth envelope, the rapid potential oscillations are taken to have a harmonic shape, thus, $v(s)=\cos (s)$ and its antiderivative $g(s)=\sin (s)$ with $\left\langle g^{2}\right\rangle=1 / 2$. For the sake of convenience, the envelope function is described by $\Phi(x)=$ $a \operatorname{sech}(x)$. Then the potential well has a characteristic

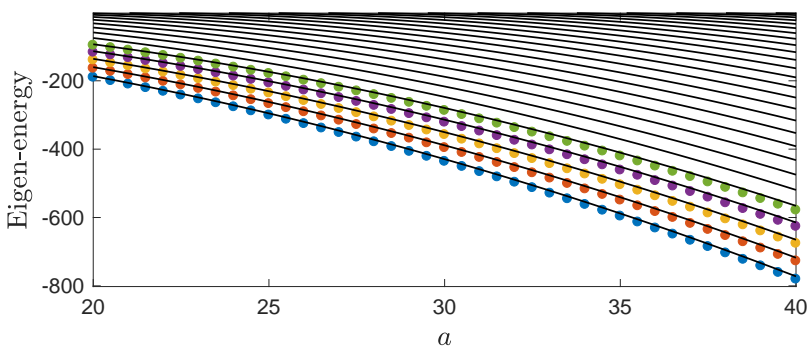

FIG. 2. (Color online) The dependence of the lowest eigenenergies of the bound states on the depth of the potential well $a$ for fixed $k=250$. Solid curves are drawn from Eq. (26), while the circles are calculated numerically using the original Hamiltonian (6).

width of the order of unity, and the effective Schrödinger equation for the averaged wave function

$$
\bar{E} \bar{\psi}(x)=\left[-\frac{d^{2}}{d x^{2}}-\frac{a^{2}}{2} \operatorname{sech}^{2}(x)\right] \bar{\psi}(x),
$$

is solvable analytically. The eigenvalue problem (25) is known as the Pöschl-Teller problem [24], and the spectrum of the bound states is given by

$$
\bar{E}_{n}=-(\lambda-n)^{2} \text {. }
$$

Here, the parameter $\lambda=\left(\sqrt{1+2 a^{2}}-1\right) / 2$ is a function of the depth $a$ and its integer part $\lfloor\lambda\rfloor$ (i.e., the largest integer smaller than or equal to $\lambda$ ) is equal to the number of bound states plus one. The index $n=0,1, \ldots,\lfloor\lambda\rfloor-$ $1,\lfloor\lambda\rfloor$ labels the bound states.

The comparison of the first five eigenfunctions of the original and the effective Hamiltonians is represented in Fig. 1. For convenience we choose the value of $a$ such that $\lambda$ becomes an integer number. In that case the eigenfunctions $\bar{\psi}(x) \sim P_{\lambda}^{\lambda-n}(\tanh (x))$, where $P_{\lambda}^{\beta}(y)$ are the associated Legendre polynomials which are related to the ordinary Legendre polynomials $P_{\lambda}(y)$ as

$$
P_{\lambda}^{\beta}(y)=(-1)^{\beta}\left(1-y^{2}\right)^{\beta / 2} \frac{d^{\beta}}{d y^{\beta}} P_{\lambda}(y) .
$$

In Fig. 2, we plot dependence of the eigenenergies on the potential amplitude parameter $a$. As one can see in Figs. 1 and 2, the approximate expression obtained using the effective Hamiltonian shows good coincidence with results of numerical calculations.

To give an example of a piecewise continuous envelope, we consider the envelope function of the form of a square barrier:

$$
\Phi(x)= \begin{cases}a, & \text { for }|x|<1 \\ 0, & \text { for }|x|>1\end{cases}
$$

According to Eq. (24), the effective potential (21) reads

$$
\begin{aligned}
V_{\mathrm{eff}}(x)= & -\left\langle g^{2}\right\rangle \Phi^{2}(x) \\
& +a\left[g\left(\varphi_{1}\right) \delta(x-1)-g\left(\varphi_{-1}\right) \delta(x+1)\right],
\end{aligned}
$$




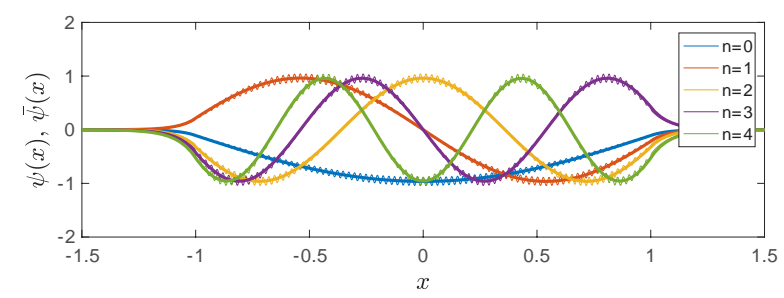

FIG. 3. (Color online) The case of piecewise continuous envelope (28). The five lowest-energy states calculated numerically using the original Hamiltonian (6) (thin oscillating curves) and found semi-analytically for the effective potential (29) (thick curves). The parameters are set to $a=20$ and $k \approx 250$ is chosen in such a way that $g\left(\varphi_{-1,1}\right)$ vanishes.

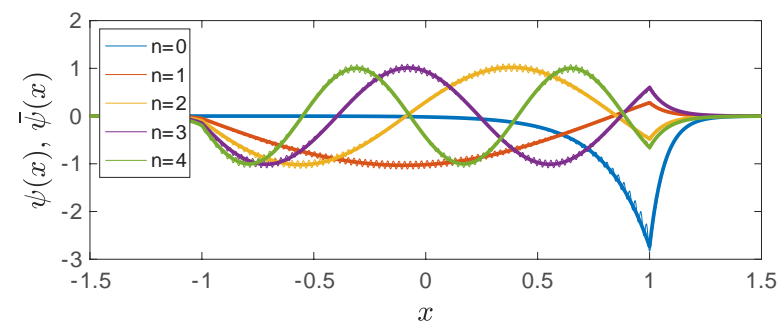

FIG. 4. (Color online) The case of piecewise continuous envelope (28). The five lowest-energy states calculated numerically using the original Hamiltonian (6) (thin oscillating curves) and found semi-analytically for the effective potential (29) (thick curves). The parameters are set to $a=20$ and $k \approx 250$ is chosen in such a way that $g\left(\varphi_{-1,1}\right)=-1$.

with $\varphi_{ \pm 1}= \pm 1 \cdot k$, and features two delta-function terms at the points of discontinuity.

We perform numerical simulations with high frequency profile function $v(s)=\cos (s)$ which gives $g(s)=\sin (s)$. We choose an appropriate value of $k$ to ensure $\varphi_{ \pm 1}=0$, and the effective potential (29) has the shape of an ordinary symmetric quantum well of a finite depth $a^{2} / 2$. The comparison of the first five eigenfunctions of the original and the effective Hamiltonian is presented in Fig. 3. We note an excellent agreement of the two sets of results, which demonstrates the applicability of the approach.

In order to demonstrate the asymmetric and singular case, we use $v(s)=\sin (s)$ whose antiderivative is $g(s)=-\cos (s)$. Again, we set $\varphi_{ \pm 1}=0$, but now the effective potential (29) has the shape of a square quantum well with two extra delta-function peaks situated at the edges: A repulsive (resp. attractive) delta-function peak of strength $a$ is centered at $x=-1$ (resp. $x=1)$. Fig. 4 again shows an excellent agreement between the exact numerical calculation and the results obtained using the effective potential. As expected from Eq. (29), the probability density is increased close to the right side of the potential well, i.e. at the position of the attractive delta-function singularity.

\section{OVERLAPPING LASER BEAMS}

The preceding analysis shows that the case of oscillating potentials with a zero mean is much more intriguing than that with a finite mean. In this subsection, we describe a practical setup that can be straightforwardly realized for ultracold atoms moving in optical lattices.

We start with two coherent laser beams, red-detuned from the atomic resonance, polarized in the same (e.g., vertical) direction and intersecting at an acute angle $2 \alpha$, as shown in Fig. 5. We assume that the beams are characterized by the angular frequency $\omega$, the wavenumber $\kappa$, and the cross-sectional intensity profile is described by a Gaussian function of width $b$, i.e.,

$$
I=I_{0} \exp \left(-\frac{r_{\perp}^{2}}{2 b^{2}}\right)
$$

here, $r_{\perp}$ is distance of a given point from the central axis of the beam. Let us consider the setup where an external trapping potential restricts the motion of ultracold atoms in the vicinity of the $x$ axis. Near the $x$ axis the respective electric fields created by the two beams are given by

$$
\begin{aligned}
& E_{1}=E_{0} \cos (\omega t-\kappa x \cos \alpha) \exp \left[-\frac{x^{2} \sin ^{2} \alpha}{2 b^{2}}\right], \\
& E_{2}=E_{0} \cos (\omega t+\kappa x \cos \alpha) \exp \left[-\frac{x^{2} \sin ^{2} \alpha}{2 b^{2}}\right] .
\end{aligned}
$$

The resulting intensity distribution is given by the timeaveraged square of the total electric field $E_{1}+E_{2}$. Thus

$$
\begin{aligned}
I & \sim 2 E_{0}^{2} \cos ^{2}(\kappa x \cos \alpha) \exp \left[-\frac{x^{2} \sin ^{2} \alpha}{b^{2}}\right] \\
& =E_{0}^{2}\{1+\cos (2 \kappa x \cos \alpha)\} \exp \left[-\frac{x^{2} \sin ^{2} \alpha}{b^{2}}\right],
\end{aligned}
$$

and the resulting intensity distribution creates a rapidly oscillating potential profile with a slowly-varying Gaussian envelope of a characteristic width $b / \sin \alpha$ which may be much larger than the wavelength. Although this potential does not have a zero mean, the background contribution

$$
V_{\mathrm{bg}} \sim E_{0}^{2} \exp \left[-\frac{x^{2} \sin ^{2} \alpha}{b^{2}}\right]
$$

can be canceled by applying an additional blue-detuned laser beam (see Fig. 5) polarized in the orthogonal direction and creating a broad Gaussian repulsive potential.

\section{CONCLUSIONS AND OUTLOOK}

We showed that quantum dynamics in potentials with fast spatial oscillations can be approximately described by a smoothed wave function that reproduces the overall structure of the true wave function but neglects its rapid 


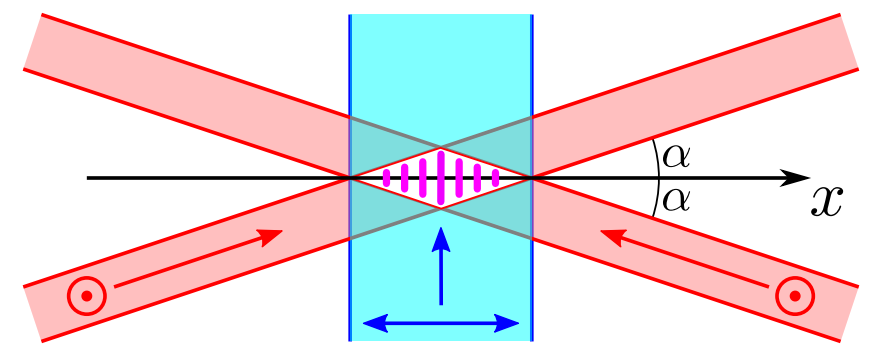

FIG. 5. (Color online) Laser-beam configuration for the creation of an oscillating potential with zero mean. Two reddetuned beams intersect at an angle and create an interference pattern. An additional blue-detuned beam is polarized in an orthogonal direction and contributes an additional repulsive profile.

small-scale oscillations. The equation of motion for the effective dynamics retains the form of the Schrödinger equation (20) and can be analyzed based on the usual framework and intuition available in single-particle quantum mechanics. In particular, generalizations to fewor many-particle problems can be readily made. In this context, it is intuitively clear that inter-particle interactions will not be modified at large length scales, however, at short scales - comparable to the range of the performed averaging - interesting modifications may take place and are worth to be investigated in future work.

\section{ACKNOWLEDGMENTS}

This research was supported by the Lithuanian Research Council under grant No. APP-4/2016.

\section{Appendix: Alternative derivation of the effective Hamiltonian}

Here we derive the effective Schrödinger equation (20) for quantum dynamics in rapidly oscillating spatial potentials using an alternative approach, inspired by an analogous treatment of classical motion in the presence of a rapidly oscillating force [25].

Our task is to approximately solve the Schrödinger equation

$$
E \psi(x)=-\frac{d^{2}}{d x^{2}} \psi(x)+[k v(k x) \Phi(x)+\rho(x)] \psi(x),
$$

with a slowly varying envelope function $\Phi(x)$ and a rapidly oscillating function $v(s)$ for a large but finite $k$. Anticipating the result obtained in the main text, we represent the wave function as a sum

$$
\psi(x)=\bar{\psi}(x)+\xi(x),
$$

of the smoothed wave function $\bar{\psi}(x)$ and a correction $\xi(x)$ that scales as $k^{-1}$. Substituting the wave function (A.2) into the Schrödinger equation (A.1) we obtain

$$
E \bar{\psi}+E \xi=-\frac{d^{2}}{d x^{2}} \bar{\psi}-\frac{d^{2}}{d x^{2}} \xi+k \Phi v \bar{\psi}+k \Phi v \xi+\rho \bar{\psi}+\rho \xi,
$$

which separates into two equations for oscillatory and smooth terms, respectively. Focusing first on the oscillating part

$$
E \xi=-\frac{d^{2}}{d x^{2}} \xi+k \Phi v \bar{\psi}+k \Phi v \xi+\rho \xi
$$

we collect the terms that are of the order of $k$ and arrive at a differential equation for the correction $\xi(x)$

$$
0=-\frac{d^{2}}{d x^{2}} \xi+k \Phi v \bar{\psi}
$$

Assuming that $\Phi$ and $\bar{\psi}$ change slowly, the solution can be written as

$$
\xi(x)=k^{-1} \Phi(x) \bar{\psi}(x) w(k x),
$$

with $w^{\prime \prime}(s)=v(s)$; here, $w(s)$ is also a periodic function with zero mean. Averaging Eq. (A.3) over one spatial period we obtain

$$
E \bar{\psi}=-\frac{d^{2}}{d x^{2}} \bar{\psi}+k \Phi \overline{v \xi}+\rho \bar{\psi} .
$$

Let us evaluate the average $\overline{v \xi}$ in the second term on the right-hand side. Using Eq. (A.6) we get

$$
\overline{v \xi}=k^{-1} \Phi \bar{\psi}\langle v w\rangle=-k^{-1} \Phi \bar{\psi}\left\langle\left(w^{\prime}\right)^{2}\right\rangle .
$$

This leads to the result, equivalent to Eq. (20) since $w^{\prime}(s)$ is the antiderivative of $v(s)$, which is denoted $g(s)$ in the main text.
[1] M. Born and R. Oppenheimer, Annalen der Physik 389, 457 (1927).

[2] W. Kołsos, Advances in Quantum Chemistry 5, 99 (1970).

[3] A. Eckardt, Rev. Mod. Phys. 89, 011004 (2017).
[4] C. Gross and I. Bloch, Science 357, 995 (2017).

[5] M. Aidelsburger, M. Atala, M. Lohse, J. T. Barreiro, B. Paredes, and I. Bloch, Phys. Rev. Lett. 111, 185301 (2013).

[6] H. Miyake, G. A. Siviloglou, C. J. Kennedy, W. C. Bur- 
ton, and W. Ketterle, Phys. Phys. Lett. 111, 185302 (2013).

[7] M. Atala, M. Aidelsburger, M. Lohse, J. T. Barreiro, B. Paredes, and I. Bloch, Nat. Phys. 10, 588 (2014).

[8] M. Aidelsburger, M. Lohse, C. Schweizer, M. Atala, J. T. Barreiro, S. Nascimbène, N. R. Cooper, I. Bloch, and N. Goldman, Nat. Phys. 11, 162 (2015).

[9] C. J. Kennedy, W. C. Burton, W. C. Chung, and W. Ketterle, Nat. Phys. 11, 859 (2015).

[10] M. E. Tai, A. Lukin, M. Rispoli, R. Schittko, T. Menke, D. Borgnia, P. M. Preiss, F. Grusdt, A. M. Kaufman, and M. Greiner, Nature 546, 519 (2017).

[11] N. Goldman and J. Dalibard, Phys. Rev. X 4, 031027 (2014).

[12] A. Eckardt and E. Anisimovas, New J. Phys. 17, 093039 (2015).

[13] V. Novičenko, E. Anisimovas, and G. Juzeliūnas, Phys. Rev. A 95, 023615 (2017).

[14] G. Jotzu, M. Messer, R. Desbuquois, M. Lebrat, T. Uehlinger, D. Greif, and T. Esslinger, Nature 515, 237 (2014).

[15] A. G. Grushin, A. Gómez-León, and T. Neupert, Phys. Rev. Lett. 112, 156801 (2014).
[16] P. Windpassinger and K. Sengstock, Rep. Progr. Phys. 76, 086401 (2013).

[17] E. Witten, Nucl. Phys. B 188, 513 (1981).

[18] F. Cooper, A. Khare, and U. Sukhatme, Physics Reports 251, 267 (1995).

[19] F. Cooper, A. Khare, and U. Sukhatme, Supersymmetry in quantum mechanics (World Scientific, Singapore, 2001).

[20] A. Gangopadhyaya, J. V. Mallow, and C. Rasinariu, Supersymmetric quantum mechanics: an introduction (World Scientific, Singapore, 2018).

[21] D. E. Ruiz and I. Y. Dodin, Phys. Rev. A 95, 032114 (2017).

[22] V. Burd, Method of Averaging for Differential Equations on an Infinite Interval (Taylor \& Francis Group, 2007).

[23] J. A. Sanders, F. Verhulst, and J. Murdock, Averaging Methods in Nonlinear Dynamical Systems (Springer, 2007).

[24] G. Pöschl and E. Teller, Z. Phys. 83, 143 (1933).

[25] L. D. Landau and E. M. Lifshitz, Mechanics, 3rd ed., Course of Theoretical Physics, Vol. 1 (ButterworthHeinemann, Oxford, 1976). 